\title{
Hydrophobic Association in Mixed Urea-TMAO Solutions
}

\author{
Pritam Ganguly ${ }^{1,2}$, Nico F. A. van der Vegt ${ }^{3}$, and Joan-Emma Shea*1,2 \\ ${ }^{1}$ Department of Chemistry and Biochemistry, University of California at Santa \\ Barbara, Santa Barbara, California 93106, USA. \\ ${ }^{2}$ Department of Physics, University of California at Santa Barbara, Santa \\ Barbara, California 93106, USA. \\ ${ }^{3}$ Eduard-Zintl-Institut für Anorganische und Physikalische Chemie and Center of \\ Smart Interfaces, Technische Universität Darmstadt, Alarich-Weiss-Straße 10, \\ Darmstadt 64287, Germany.
}

${ }^{*}$ Corresponding author. E-mail: shea@chem.ucsb.edu 
Force-field parameters for TMAO: The force-field parameters for the TMAO models are listed below. The combination rules used for the Lennard-Jones cross-interactions are as follows: $\sigma_{i j}=\sqrt{\sigma_{i} \sigma_{j}}, \epsilon_{i j}=\sqrt{\epsilon_{i} \epsilon_{j}}$ for the Kast, ${ }^{1}$ the Netz, ${ }^{2}$ the Hölzl, ${ }^{3}$ and the Shea models ${ }^{4}$ and $\sigma_{i j}=$ $\left(\sigma_{i}+\sigma_{j}\right) / 2, \epsilon_{i j}=\sqrt{\epsilon_{i} \epsilon_{j}}$ for the Garcia model. ${ }^{5}$ The TMAO-TMAO Lennard-Jones interactions are scaled as $\epsilon_{i j}=0.75 \sqrt{\epsilon_{i} \epsilon_{j}}$ for the Garcia model. ${ }^{5}$

\begin{tabular}{|c|c|c|c|c|}
\hline Force field & Atom & $\sigma(\mathbf{n m})$ & $\epsilon\left(\mathbf{k J} \mathbf{~ m o l}^{-\mathbf{1}}\right)$ & $q(e)$ \\
\hline \multirow{4}{*}{ Kast } & C & 0.3041 & 0.2826 & -0.260 \\
\hline & $\mathrm{H}$ & 0.1775 & 0.0773 & 0.110 \\
\hline & $\mathrm{O}$ & 0.3266 & 0.6379 & -0.650 \\
\hline & $\mathrm{N}$ & 0.2926 & 0.8360 & 0.440 \\
\hline \multirow{4}{*}{ Netz } & $\mathrm{C}$ & 0.3600 & 0.2826 & -0.260 \\
\hline & $\mathrm{H}$ & 0.2101 & 0.0773 & 0.110 \\
\hline & $\mathrm{O}$ & 0.3266 & 0.6379 & -0.910 \\
\hline & $\mathrm{N}$ & 0.2926 & 0.8360 & 0.700 \\
\hline \multirow{4}{*}{ Garcia } & $\mathrm{C}$ & 0.3041 & 0.2826 & -0.312 \\
\hline & $\mathrm{H}$ & 0.1775 & 0.0773 & 0.132 \\
\hline & $\mathrm{O}$ & 0.3266 & 0.6379 & -0.780 \\
\hline & $\mathrm{N}$ & 0.2926 & 0.8360 & 0.528 \\
\hline \multirow{4}{*}{ Hölzl } & $\mathrm{C}$ & 0.3707 & 0.2830 & -0.260 \\
\hline & $\mathrm{H}$ & 0.2130 & 0.0775 & 0.110 \\
\hline & $\mathrm{O}$ & 0.3266 & 0.6389 & -0.815 \\
\hline & $\mathrm{N}$ & 0.2926 & 0.8374 & 0.605 \\
\hline \multirow{4}{*}{ Shea } & $\mathrm{C}$ & 0.3385 & 0.2800 & -0.260 \\
\hline & $\mathrm{H}$ & 0.2319 & 0.0660 & 0.110 \\
\hline & $\mathrm{O}$ & 0.3109 & 0.6040 & -0.650 \\
\hline & $\mathrm{N}$ & 0.3205 & 0.7430 & 0.440 \\
\hline
\end{tabular}

Table S1: Non-bonded interaction parameters of the atoms of a TMAO molecule for a) Kast model, ${ }^{1}$ b) Netz model, ${ }^{2}$ c) Garcia model, ${ }^{5}$ d) Hölzl model,${ }^{3}$ and e) Shea model. ${ }^{4}$ 
Bond parameters

\begin{tabular}{ccc}
\hline Bond type & Force constant $\left(\mathbf{k J ~ m o l}^{-\mathbf{1}} \mathbf{n m}^{-\mathbf{2}}\right)$ & Equilibrium bond length $\mathbf{( n m})$ \\
\hline $\mathrm{O}-\mathrm{N}$ & 143198 & 0.1407 \\
\hline $\mathrm{C}-\mathrm{N}$ & 107079 & 0.1506 \\
\hline $\mathrm{C}-\mathrm{H}$ & 247021 & 0.1082 \\
\hline
\end{tabular}

Angle parameters

\begin{tabular}{ccc}
\hline Angle type & Force constant $\left(\mathrm{kJ} \mathrm{mol}^{-\mathbf{1}} \mathbf{~ r a d}^{-\mathbf{2}}\right)$ & Equilibrium angle (deg) \\
\hline $\mathrm{O}-\mathrm{N}-\mathrm{C}$ & 254.7 & 109.99 \\
\hline $\mathrm{N}-\mathrm{C}-\mathrm{H}$ & 208.7 & 108.07 \\
\hline
\end{tabular}

Dihedral parameters

\begin{tabular}{cccc}
\hline Dihedral type & Well depth $\left(\mathbf{k J ~ m o l}^{-\mathbf{1}}\right)$ & Phase (deg) & Multiplicity \\
\hline O-N-C-H & 1.129 & 0 & 3
\end{tabular}

Table S2: Bonded interaction parameters of the TMAO molecules for the Kast model. Other TMAO models use the same bonded parameters.

\section{Spatial distribution of urea around neopentane:}

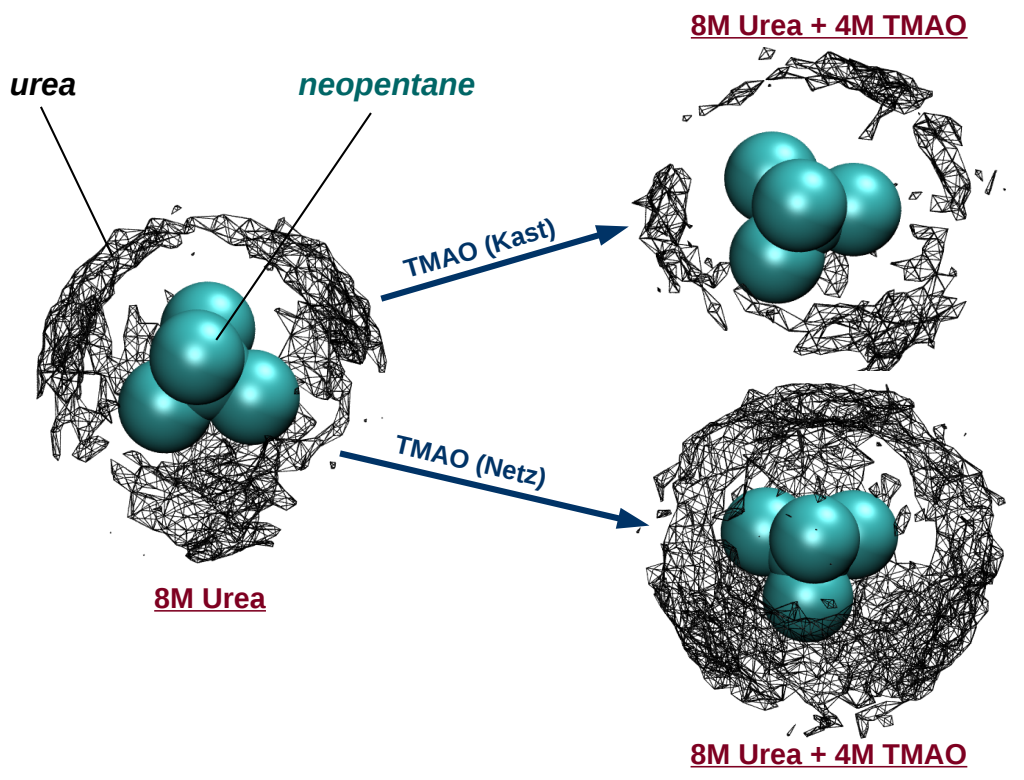

Figure S1: Spatial density maps of urea around neopentane obtained with the Kast and the Netz TMAO force fields. Shown are the urea distributions with density 2 times or higher than the bulk urea density. The Kast TMAO model depletes urea whereas the Netz model enhances urea-neopentane association. Figures are rendered using Visual Molecular Dynamics (VMD) software. ${ }^{6}$ 
Alanine-urea KBIs:

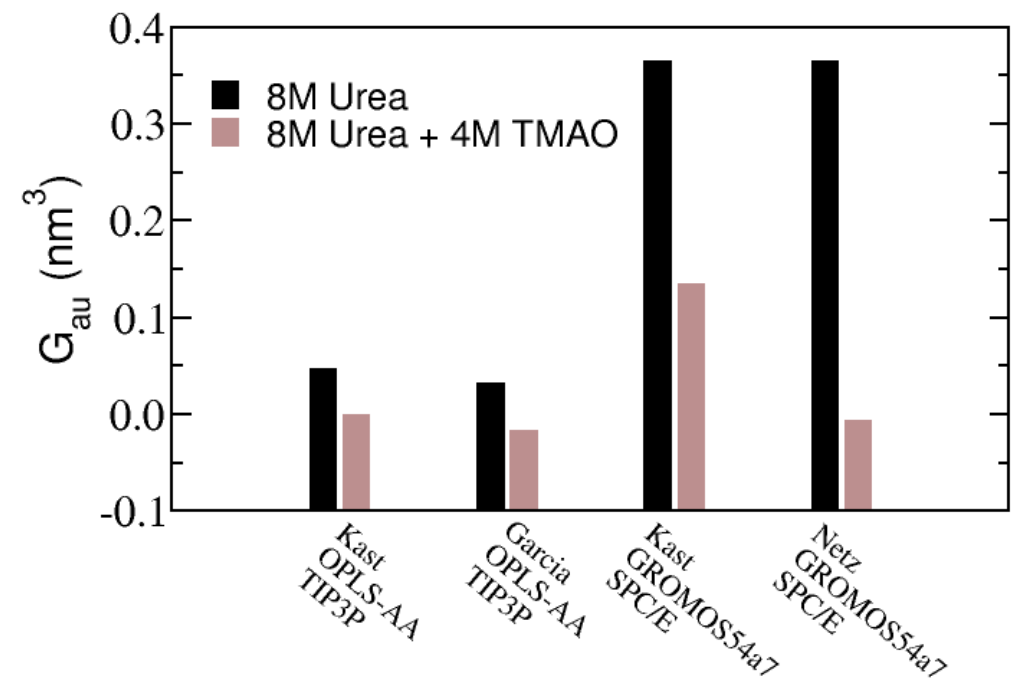

Figure S2: Comparison of alanine-urea Kirkwood-Buff integrals $\left(G_{a u}\right)$ obtained with the Kast, ${ }^{1}$ the Netz $^{2}$ and the Garcia ${ }^{5}$ TMAO force fields. Shown are the data for alanine in $8 \mathrm{M}$ urea and in the mixture of $8 \mathrm{M}$ urea and $4 \mathrm{M}$ TMAO. For alanine GROMOS54A7 $7^{7}$ and OPLS-AA ${ }^{8}$ models are used. Water was modeled with SPC/E ${ }^{9}$ and TIP3P. ${ }^{10}$ Combination rules for Lennard-Jones cross-interactions: $\sigma_{i j}=\sqrt{\sigma_{i} \sigma_{j}}$ for Kast and Netz labels and $\sigma_{i j}=\left(\sigma_{i}+\sigma_{j}\right) / 2$ for Garcia labels. This figure partially uses data from the reference: J. Phys. Chem. Lett. 2015, 6, 581. 


\section{Urea-water KBIs:}

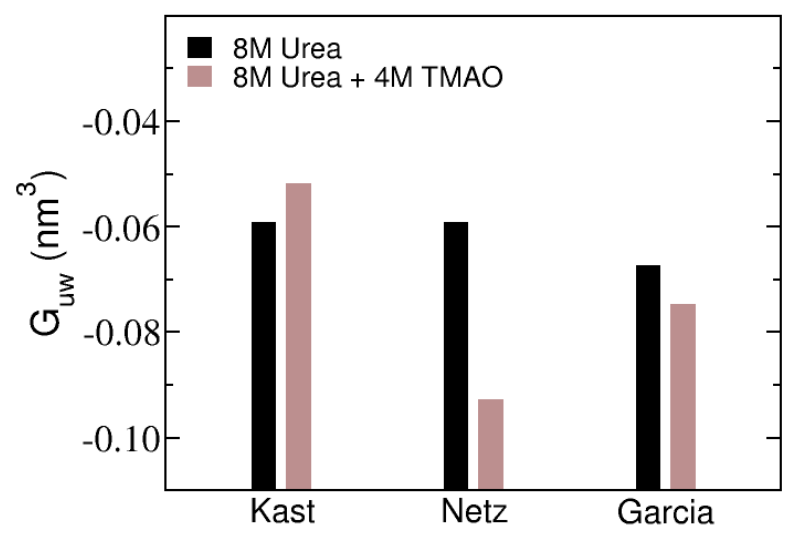

Figure S3: Urea-water Kirkwood-Buff integrals $\left(\mathrm{G}_{\mathrm{uw}}\right)$ obtained with the Kast, the Netz and the Garcia TMAO force fields. Shown are the data for neopentane in $8 \mathrm{M}$ urea and in the mixture of $8 \mathrm{M}$ urea and 4 M TMAO.

\section{TMAO-water KBIs:}

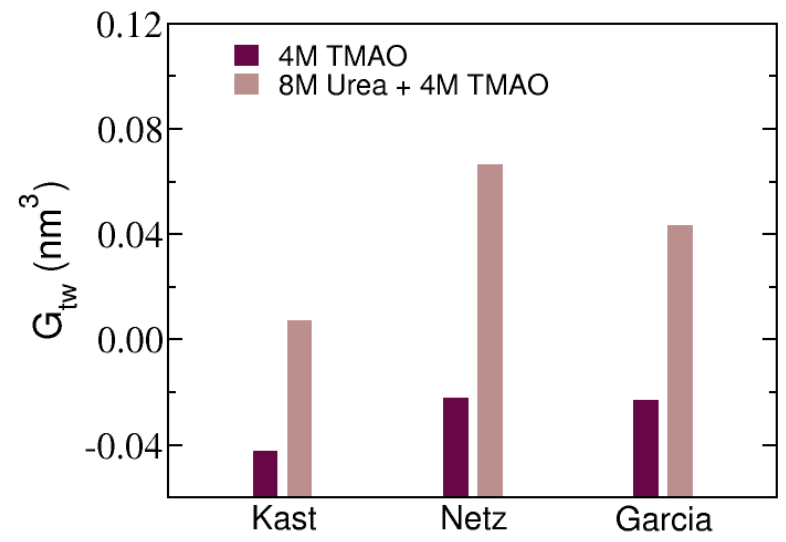

Figure S4: TMAO-water Kirkwood-Buff integrals $\left(\mathrm{G}_{\mathrm{tw}}\right)$ obtained with the Kast, the Netz and the Garcia TMAO force fields. Shown are the data for neopentane in $4 \mathrm{M}$ TMAO and in the mixture of $8 \mathrm{M}$ urea and 4 M TMAO. 


\section{Urea-urea KBIs:}

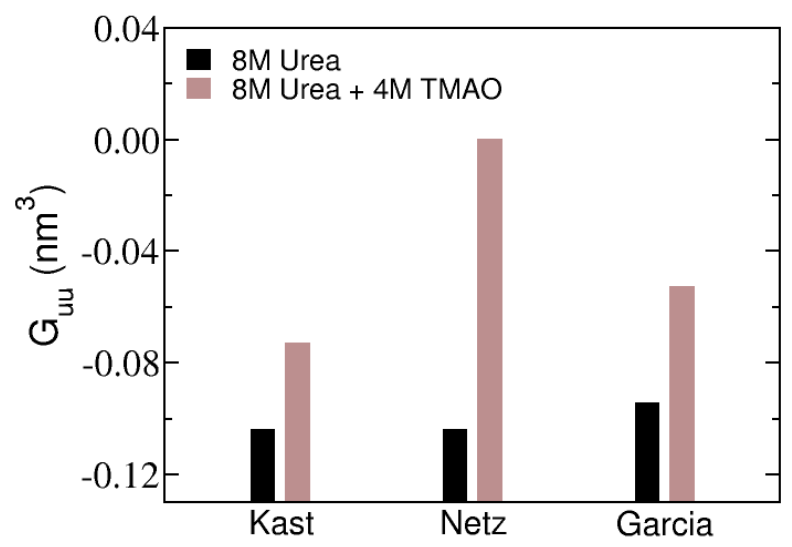

Figure S5: Urea-urea Kirkwood-Buff integrals $\left(\mathrm{G}_{\mathrm{uu}}\right)$ obtained with the Kast, the Netz and the Garcia TMAO force fields. Shown are the data for neopentane in $8 \mathrm{M}$ urea and in the mixture of $8 \mathrm{M}$ urea and 4 M TMAO.

TMAO-TMAO KBIs:

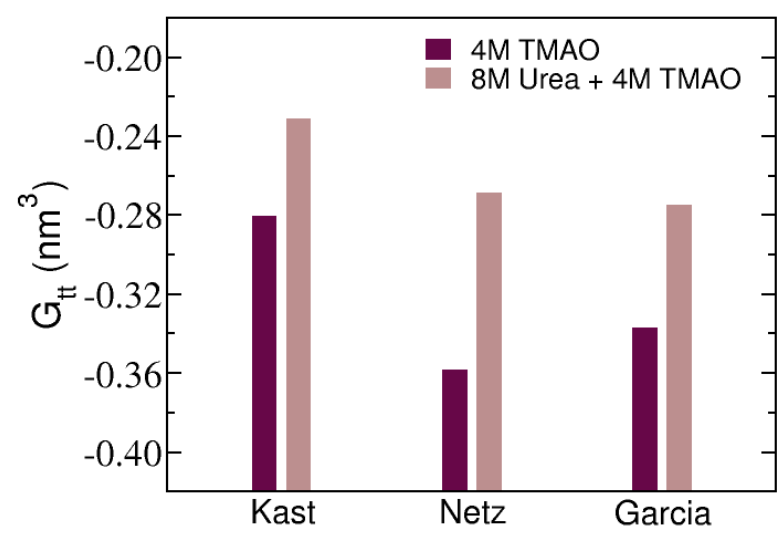

Figure S6: TMAO-TMAO Kirkwood-Buff integrals $\left(\mathrm{G}_{\mathrm{tt}}\right)$ obtained with the Kast, the Netz and the Garcia TMAO force fields. Shown are the data for neopentane in $4 \mathrm{M}$ TMAO and in the mixture of $8 \mathrm{M}$ urea and 4 M TMAO. 
TMAO-water and TMAO-urea hydrogen bonds:

\begin{tabular}{ccccc}
\hline Force field & 4M TMAO & \multicolumn{4}{c}{ 8M Urea + 4M TMAO } \\
\hline & $\mathrm{H}_{\mathrm{tw}}$ & $\mathrm{H}_{\mathrm{tw}}$ & $\mathrm{H}_{\mathrm{tu}}$ & $\mathrm{H}_{\mathrm{tw}}+\mathrm{H}_{\mathrm{tu}}$ \\
\hline Kast & 2.65 & 1.72 & 1.06 & 2.78 \\
\hline Netz & 3.16 & 2.53 & 0.74 & 3.27 \\
\hline Garcia & 3.16 & 2.35 & 0.89 & 3.24 \\
\hline
\end{tabular}

Table S3: Shown are the average number of TMAO-water $\left(\mathrm{H}_{\mathrm{tw}}\right)$ and TMAO-urea $\left(\mathrm{H}_{\mathrm{tu}}\right)$ hydrogen bonds per TMAO molecule in $4 \mathrm{M}$ TMAO solutions and in mixed $8 \mathrm{M}$ urea and $4 \mathrm{M}$ TMAO solution containing neopentane.

Simulation results with Hölzl and Shea models:

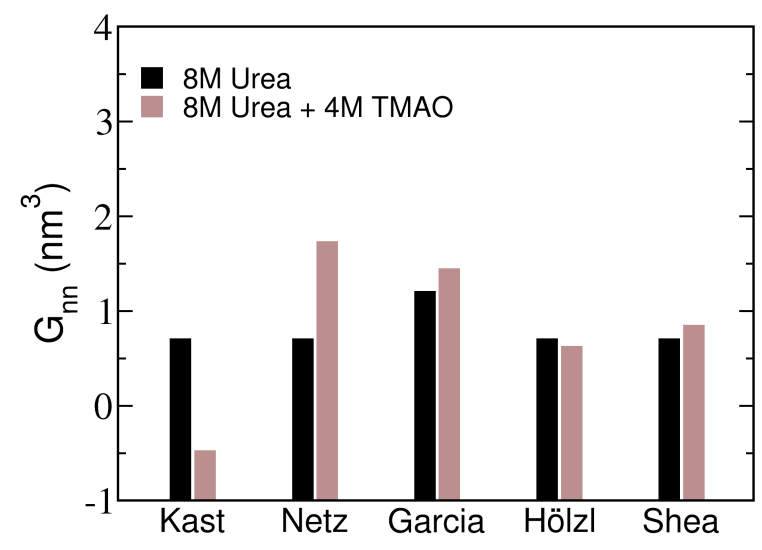

Figure S7: Neopentane-neopentane Kirkwood-Buff integrals $\left(\mathrm{G}_{\mathrm{nn}}\right)$ obtained with the Kast, the Netz, the Garcia, the Hölzl, and the Shea TMAO force fields. Shown are the data for neopentane in $8 \mathrm{M}$ urea and in the mixture of $8 \mathrm{M}$ urea and $4 \mathrm{M}$ TMAO. 


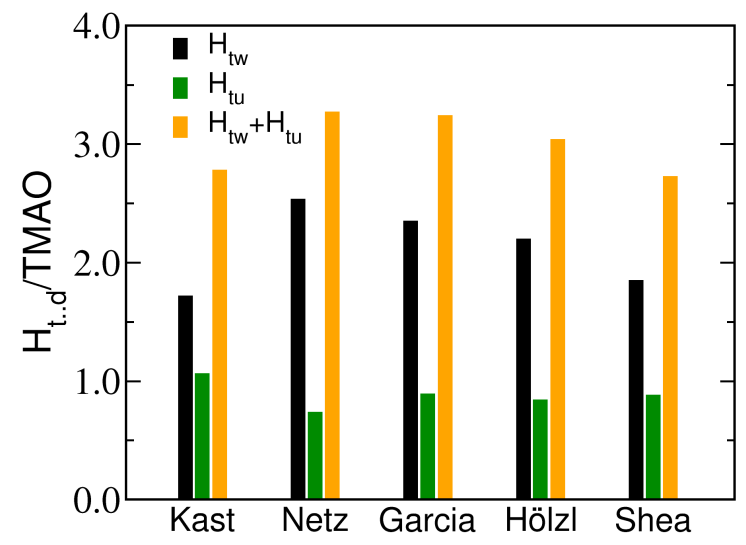

Figure S8: Comparison of hydrogen-bonding propensity of TMAO with water and urea for the Kast, the Netz, the Garcia, the Hölzl, and the Shea TMAO force fields. Shown are the data for neopentane in the mixture of $8 \mathrm{M}$ urea and $4 \mathrm{M}$ TMAO.

Comparison of the combination rules for the Lennard-Jones interactions: We have chosen SPC/E water model and the combination rule as $\sigma_{i j}=\sqrt{\sigma_{i} \sigma_{j}}$ for the Kast and the Netz models because the urea model ${ }^{11}$ was originally developed using these parameters and it showed correct variation of the activity coefficient of binary urea-water solutions when compared to the experiments. However, the Garcia model was developed using TIP3P water and the combination rule $\sigma_{i j}=\left(\sigma_{i}+\sigma_{j}\right) / 2$ and it captured the experimental osmotic coefficients of binary TMAO-water mixtures. This justifies our choice of the water models and the combination rules for the different TMAO models. We note that in the respective original papers the Kast ${ }^{1}$ and the Netz ${ }^{2}$ models had used the combination rule of $\sigma_{i j}=\left(\sigma_{i}+\sigma_{j}\right) / 2$. In order to check the effects of the different combination rules on our results we have rerun the simulations of neopentane in $8 \mathrm{M}$ urea and in mixed $8 \mathrm{M}$ urea and $4 \mathrm{M}$ TMAO using the Kast TMAO model. Simulations with both the combination rules have shown qualitatively similar results where neopentane-neopentane and neopentane-urea aggregations are reduced when $4 \mathrm{M}$ TMAO is added to $8 \mathrm{M}$ urea solutions. However, the results obtained with the different combination rules are quantitatively different where the combination rule $\sigma_{i j}=\left(\sigma_{i}+\sigma_{j}\right) / 2$ shows moderately higher neopentane-neopentane and neopentane-urea aggregations than the combination rule $\sigma_{i j}=\sqrt{\sigma_{i} \sigma_{j}}$. The corresponding plots are shown below. 

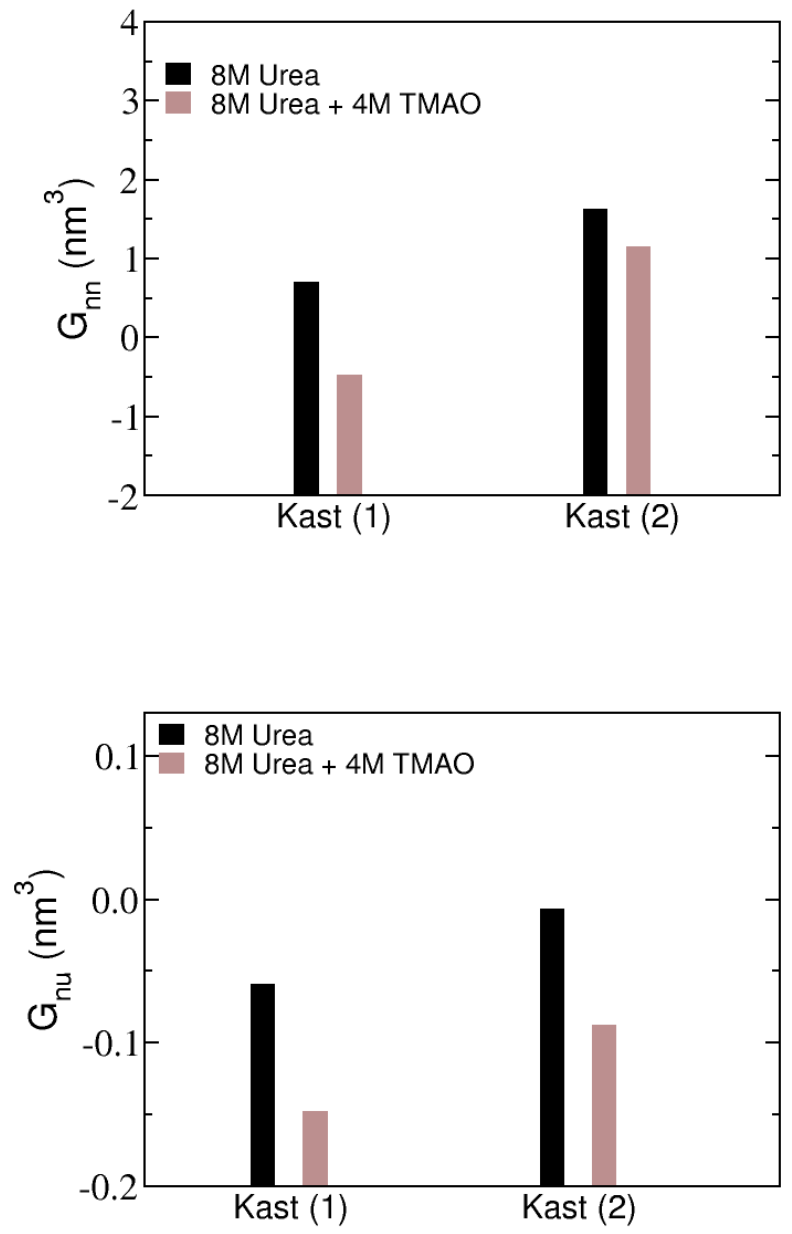

Figure S9: Upper panel: neopentane-neopentane KBIs $\left(\mathrm{G}_{\mathrm{nn}}\right)$ for neopentane in $8 \mathrm{M}$ urea and mixed $8 \mathrm{M}$ urea and $4 \mathrm{M}$ TMAO solutions. Lower panel: KBIs between neopentane and urea $\left(\mathrm{G}_{\mathrm{nu}}\right)$ for neopentane in $8 \mathrm{M}$ urea and mixed $8 \mathrm{M}$ urea and $4 \mathrm{M}$ TMAO solutions. Combination rules for Lennard-Jones crossinteractions: results with $\sigma_{i j}=\sqrt{\sigma_{i} \sigma_{j}}$ and $\epsilon_{i j}=\sqrt{\epsilon_{i} \epsilon_{j}}$ are denoted by Kast (1) label and results with $\sigma_{i j}=\left(\sigma_{i}+\sigma_{j}\right) / 2$ and $\epsilon_{i j}=\sqrt{\epsilon_{i} \epsilon_{j}}$ are denoted by Kast (2) label. 


\section{References}

[1] Kast, K. M.; Brickmann, J.; Kast, S. M.; Berry, R. S. Binary Phases of Aliphatic N-Oxides and Water: Force Field Development and Molecular Dynamics Simulation. J. Phys. Chem. A 2003, 107, 5342-5351.

[2] Schneck, E.; Horinek, D.; Netz, R. R. Insight into the Molecular Mechanisms of Protein Stabilizing Osmolytes from Global Force-Field Variations. J. Phys. Chem. B 2013, 117, 83108321.

[3] Hölzl, C.; Kibies, P.; Imoto, S.; Frach, R.; Suladze, S.; Winter, R.; Marx, D.; Horinek, D.; Kast, S. M. Design Principles for High-pressure Force Fields: Aqueous TMAO Solutions from Ambient to Kilobar Pressures. J. Chem. Phys. 2016, 144, 144104.

[4] Larini, L.; Shea, J.-E. Double Resolution Model for Studying TMAO/Water Effective Interactions. J. Phys. Chem. B 2013, 117, 13268-13277.

[5] Canchi, D. R.; Jayasimha, P.; Rau, D. C.; Makhatadze, G. I.; García, A. E. Molecular Mechanism for the Preferential Exclusion of TMAO from Protein Surfaces. J. Phys. Chem. B 2012, 116, 12095-12104.

[6] Humphrey, W., Dalke, A. and Schulten, K. VMD - Visual Molecular Dynamics. J. Molec. Graphics. 1996, 14, 33-38.

[7] Schmid, N.; Eichenberger, A. P.; Choutko, A.; Riniker, S.; Winger, M.; Mark, A. E.; van Gunsteren, W. F. Definition and Testing of the GROMOS Force-field Versions 54 A7 and 54B7. Eur. Biophys. J. 2011, 40, 843-856.

[8] Jorgensen, W. L.; Maxwell, D. S.; Tirado-Rives, J. Development and Testing of the OPLS All-Atom Force Field on Conformational Energetics and Properties of Organic Liquids. J. Am. Chem. Soc. 1996, 118, 11225-11236.

[9] Berendsen, H. J. C.; Grigera, J. R.; Straatsma, T. P. The Missing Term in Effective Pair Potentials. J. Phys. Chem. 1987, 91, 6269-6271.

[10] Jorgensen, W. L.; Chandrasekhar, J.; Madura, J. D.; Impey, R. W.; Klein, M. L. Comparison of Simple Potential Functions for Simulating Liquid Water. J. Chem. Phys. 1983, 79, 926.

[11] Weerasinghe, S.; Smith, P. E. A Kirkwood-Buff Derived Force Field for Mixtures of Urea and Water. J. Phys. Chem. B 2003, 10\%, 3891-3898. 